\title{
Special relativity on the light-front
}

\author{
Jorge Henrique Sales ${ }^{* 10}$, A. T. Suzuki ${ }^{2}$, Gislan S. Santos ${ }^{3}$, Daykson N. Possidonio \\ ${ }^{1}$ Universidade Estadual de Santa Cruz, 45662-000, Ilhéus, BA, Brasil. \\ ${ }^{2}$ La Sierra University, Physics Department, Riverside, CA 92505, United States. \\ ${ }^{3}$ Instituto Federal da Bahia, 45078-900, Vitória da Conquista, BA, Brasil.
}

Received on January 28, 2021. Revised on June 02, 2021. Accepted on June 03, 2021.

\begin{abstract}
P.A.M. Dirac in 1949 showed that it is possible to construct relativistic dynamic forms starting from the description of the initial state of a given relativistic system in any space-time surface whose distances between two points on this hypersurface has no causal connection. The dynamic evolution corresponds to such a system following a trajectory through this hypersurface. For example, the commonest hypersurface of time $t=0$ is our three-dimensional (Euclidean) space. It is invariant by rotations and translations. However, in any transformation of inertial frame of references that involves "boosts", the time coordinate is modified and, consequently, the hypersurface at $t=0$. Other hypersurfaces may be invariant through some kind of "boost"; the hyperplane that is called null-plane is such a hyperplane, defined by $x^{+}=t+z / c$, in which $c$ is the speed of light in vacuum, and plays the role of the "time" coordinate in the light-front. The null-plane defined in such a way guarantees that a "boost" in the $z$ direction does not modify the null-plane. Our aim here is to study special relativity under such a transformation of frame of references and see the consequences thereof.
\end{abstract}

Keywords: Minkowski space, Lorentz transformations, Quantum propagator.

\section{Introduction}

In relativity courses in general, it is studied that Lorentz transformations are defined in a space with four dimensions: three spatial and one temporal. In this work we discuss an alternative way to define Lorentz transformations in the light front, through of Minkowski space and its properties. This work is accessible to undergraduate physics students who have attended a course in quantum mechanics and special relativity and want to explore these themes in another proposal, such as coordinating information in the light front.

In classical physics, the time coordinate is not affected by a transformation carried out from one inertial frame to another one. The time coordinate $t$ of an inertial frame does not depend on the spatial coordinates $x, y, z$ of another frame of reference, that is, the transformation equation for the time coordinate is simply $t^{\prime}=t$. This is no longer true in special relativity, where time and space are dependent on each other. The time coordinate in one inertial reference frame depends both on the time and space coordinates of another inertial reference frame. For example, the time coordinate in the primed frame that moves along the $x$ - and $x^{\prime}$-axis with a relative speed $v$ reads now

$$
t^{\prime}=\frac{t-\frac{v}{c^{2}} x}{\sqrt{1-\frac{v^{2}}{c^{2}}}}
$$

\footnotetext{
${ }^{*}$ Correspondence email address: jhosales@uesc.br
}

Thus, instead of treating space and time separately as in the classical theory was appropriate to do so, it is now natural to treat them simultaneously. H. Minkowski [1] was the first one to clearly show how this could be done.

In special relativity then we have to abandon this idea of an absolute time and start considering the proper time for each inertial frame of reference. As a consequence what emerges is a space-time or a four-dimensional continuum, in which the time is considered as the fourth dimension. Although other metrics can be considered in such a space, we can use a metric which resembles the Euclidean metric, in everything similar to the threedimensional metric of the ordinary space. This fourdimensional structure is called Minkowski universe. An event is defined by four coordinates in the Minkowski space and the distance between two events - called the universe interval - is invariant. Thus, the universe interval assumes the role of the distance between two points in the ordinary three-dimensional space.

The vectors (four-vectors) defined in the Minkowski space and which obey the Lorentz transformations are called vectors in the Minkowski universe. In this work we consider how to explore these same concepts defined in the Minkowski space on a hyperplane known as the light front. In the literature we can find different definitions for the light front which are distinguished from each other by a numerical factor, say $\alpha$ for which typical values are $\alpha=1,2, \sqrt{2}$. Then, utilizing a transformation of the Minkowski space into the generalized light front, we obtain the transformations for the differentiation operators, general metric and Jacobian for the transformation. 
Afterwards, we express the Lorentz transformations in the light front and study their consequence for particular three inertial frames of reference. Finally, we compare the Klein-Gordon equation in the Minkowski space with its light front representation and draw some conclusions.

\section{Basics on the Light-Front}

We shall start off by considering Einstein's special relativity defined in the usual Minkowski space-time and then we shall make a transformation to the light-front coordinates.

In dealing with tensor structures for a given finite dimensional space we consider linear transformations of them and intrinsec to this is the subjacent structure for the space - which by and large is a metric space, that is, a space wherein we have defined definite rules governing lengths between two pairs of neighbouring points of such a space. For all riemannian type of spaces there exists a quadratic differential form

$$
d s^{2}=g_{i j} d x^{i} d x^{j}, \quad j=1,2, \ldots, N,
$$

in which the metric elements $g_{i j}$ are in general functions of position with a subsidiary condition $\operatorname{det}\left(g_{i j}\right) \neq 0$ so as to guarantee the existence of the inverse transformation. The very structure of the quadratic form above suggests that this metric must be symmetric under the interchange of indices $(i, j)$.

Since the metric for special relativity space-time is the Minkowski metric rather than an Euclidean one, first of all we mention that the convention choice for this metric is important right from the beginning, since upon this choice will depend the set up for our transformation properties from covariant to contravariant tensor structures.

One possible choice, which we shall call Minkowski convention is

$$
g_{\mu \nu}=g^{\mu \nu}=\left(\begin{array}{cccc}
1 & 0 & 0 & 0 \\
0 & 1 & 0 & 0 \\
0 & 0 & 1 & 0 \\
0 & 0 & 0 & -1
\end{array}\right) .
$$

In this convention the time component of the four-vector $x^{\mu}, \mu=1,2,3,4$ is pure immaginary defining the fourth component of it as $x^{4} \equiv i c t$.

Another possibility is what we call the Bjorken-Drell convention - which we prefer and shall use henceforth because it is increasingly popularized by its frequent usage among field theoreticians. In the Bjorken-Drell convention we have

$$
g_{\mu \nu}=g^{\mu \nu}=\left(\begin{array}{cccc}
1 & 0 & 0 & 0 \\
0 & -1 & 0 & 0 \\
0 & 0 & -1 & 0 \\
0 & 0 & 0 & -1
\end{array}\right) .
$$

Here the four vector is given by $x^{\mu}, \mu=0,1,2,3$ and $x^{0} \equiv$ ct is the time component.
Consequently, any arbitrary (contravariant) four vector in the Bjorken-Drell convention is written down as

$$
A^{\mu}=\left(A^{0}, A^{1}, A^{2}, A^{3}\right),
$$

whereas the covariant version of it is written down as

$$
A_{\mu}=\left(A_{0},-A_{1},-A_{2},-A_{3}\right),
$$

We want to express these in terms of light-front coordinates. Dirac 2 showed that it is possible to construct dynamical forms starting from a description of the initial state of a relativistic system in any space-time surface, whose distances between points are not connected causally. The dynamical evolution corresponds to the system following a trajectory through the hypersurfaces. For example, the hypersurface $t=0$ is our threedimensional space. It is invariant under translations and rotations. However, in any transformation of inertial frame of reference that involves "boosts" the time coordinate is modified and therefore the hypersurface $t=0$. Other hypersurfaces can be invariant by some type of "boost"; it is the case of the hyperplane called light-front, defined by $x^{+}=t+z / c$, which is the "time" coordinate for the light front. The traditional light-front coordinates 35 are defined as

$$
\begin{aligned}
x^{+} & =\frac{1}{\sqrt{2}}\left(x^{0}+x^{3}\right) \\
x^{-} & =\frac{1}{\sqrt{2}}\left(x^{0}-x^{3}\right) \\
\overrightarrow{\mathbf{x}}_{\perp} & =x^{1} \hat{\imath}+x^{2} \hat{\jmath},
\end{aligned}
$$

in which the normalization factor in the first and second lines are somewhat arbitrary. Inverse transformation can be pulled out without difficulty from these:

$$
\begin{aligned}
x^{0} & =\frac{1}{\sqrt{2}}\left(x^{+}+x^{-}\right) \\
x^{1} & =x^{1} \\
x^{2} & =x^{2} \\
x^{3} & =\frac{1}{\sqrt{2}}\left(x^{+}-x^{-}\right)
\end{aligned}
$$

The Jacobian for the transformation from one to another can be evaluated through the following determinant

$$
|J|=\left|\begin{array}{llll}
\frac{\partial x^{0}}{\partial x^{+}} & \frac{\partial x^{0}}{\partial x^{-}} & \frac{\partial x^{0}}{\partial x^{1}} & \frac{\partial x^{0}}{\partial x^{2}} \\
\frac{\partial x^{1}}{\partial x^{+}} & \frac{\partial x^{1}}{\partial x^{-}} & \frac{\partial x^{1}}{\partial x^{1}} & \frac{\partial x^{1}}{\partial x^{2}} \\
\frac{\partial x^{2}}{\partial x^{+}} & \frac{\partial x^{2}}{\partial x^{-}} & \frac{\partial x^{2}}{\partial x^{1}} & \frac{\partial x^{2}}{\partial x^{2}} \\
\frac{\partial x^{3}}{\partial x^{+}} & \frac{\partial x^{3}}{\partial x^{-}} & \frac{\partial x^{3}}{\partial x^{1}} & \frac{\partial x^{3}}{\partial x^{2}}
\end{array}\right|
$$


For our present choice for the normalization factor in $x^{0}$ and $x^{3}$ (or equivalently for $x^{+}$and $x^{-}$), the Jacobian is equal to 1 . Then, for the light-front coordinates, the position four-vector is written as

$$
x_{\mathrm{LF}}^{\mu}=\left(x^{+}, x^{-}, \overrightarrow{\mathbf{x}}^{\perp}\right)
$$

\section{Generalized Light-Front}

Instead of the traditional light-front normalization factor $2^{-\frac{1}{2}}$ utilized for the plus and minus components, we introduce the generalized normalization factor $\alpha^{-1}$ :

$$
\begin{aligned}
x^{+} & =\frac{1}{\alpha}\left(x^{0}+x^{3}\right) \\
x^{-} & =\frac{1}{\alpha}\left(x^{0}-x^{3}\right) \\
\overrightarrow{\mathbf{x}}^{\perp} & =x^{1} \hat{\imath}+x^{2} \hat{\jmath}
\end{aligned}
$$

In a similar way for the conjugated moment

$$
\begin{gathered}
k^{+}=\frac{1}{\alpha}\left(k^{0}+k^{3}\right) \\
k^{-}=\frac{1}{\alpha}\left(k^{0}-k^{3}\right) \\
\overrightarrow{\mathbf{k}}^{\perp}=k^{1} \hat{\imath}+k^{2} \hat{\jmath}
\end{gathered}
$$

Clearly, equation (5) does remain invariant no matter the value of the parameter $\alpha$ that is chosen. For the coordinates that are affected, the inverse transformation becomes

$$
\begin{aligned}
& x^{0}=\frac{\alpha}{2}\left(x^{+}+x^{-}\right) \\
& x^{3}=\frac{\alpha}{2}\left(x^{+}-x^{-}\right),
\end{aligned}
$$

and

$$
\begin{aligned}
& k^{0}=\frac{\alpha}{2}\left(k^{+}+k^{-}\right) \\
& k^{3}=\frac{\alpha}{2}\left(k^{+}-k^{-}\right)
\end{aligned}
$$

Partial differentiation in Eqs. (3)-(4) with respect to $x^{+}$, yields:

$$
\begin{aligned}
& \frac{\partial x^{+}}{\partial x^{+}}=\frac{1}{\alpha} \frac{\partial}{\partial x^{+}}\left(x^{0}+x^{3}\right) \quad \Rightarrow \quad \frac{\partial x^{0}}{\partial x^{+}}+\frac{\partial x^{3}}{\partial x^{+}}=\alpha \\
& \frac{\partial x^{-}}{\partial x^{+}}=\frac{1}{\alpha} \frac{\partial}{\partial x^{+}}\left(x^{0}-x^{3}\right) \Rightarrow \frac{\partial x^{0}}{\partial x^{+}}-\frac{\partial x^{3}}{\partial x^{+}}=0
\end{aligned}
$$

By adding Eqs. 10 and (11), we get:

$$
\frac{\partial x^{0}}{\partial x^{+}}=\frac{\alpha}{2}
$$

Similarly, by subtracting Eq. (11) from Eq. (10) we get:

$$
\frac{\partial x^{3}}{\partial x^{+}}=\frac{\alpha}{2}
$$

An analogous differentiation analysis with respect to the $x^{-}$-component in Eqs. (3)-(4) leads to:

$$
\begin{aligned}
& \frac{\partial x^{0}}{\partial x^{-}}=\frac{\alpha}{2} \\
& \frac{\partial x^{3}}{\partial x^{-}}=-\frac{\alpha}{2}
\end{aligned}
$$

For a given function $f=f\left(x^{0}, x^{3}\right)$ differentiation with respect to $x^{+}$and $x^{-}$can be evaluated through linearity properties of the differentiation operator

$$
\frac{\partial f}{\partial x^{+}}=\frac{\partial f}{\partial x^{0}} \frac{\partial x^{0}}{\partial x^{+}}+\frac{\partial f}{\partial x^{3}} \frac{\partial x^{3}}{\partial x^{+}}
$$

Utilizing Eq. 12 and Eq. 13, we get:

$$
\frac{\partial f}{\partial x^{+}}=\frac{\alpha}{2}\left(\frac{\partial f}{\partial x^{0}}+\frac{\partial f}{\partial x^{3}}\right)
$$

Similarly

$$
\frac{\partial f}{\partial x^{-}}=\frac{\alpha}{2}\left(\frac{\partial f}{\partial x^{0}}-\frac{\partial f}{\partial x^{3}}\right)
$$

The corresponding inverse transformation reads:

$$
\begin{gathered}
\frac{\partial f}{\partial x^{0}}=\frac{1}{\alpha}\left(\frac{\partial f}{\partial x^{+}}+\frac{\partial f}{\partial x^{-}}\right) \\
\frac{\partial f}{\partial x^{3}}=\frac{1}{\alpha}\left(\frac{\partial f}{\partial x^{+}}-\frac{\partial f}{\partial x^{-}}\right)
\end{gathered}
$$

As expected, differentiation operator for + and components change roles when compared to the position vector + and - components:

$$
\begin{aligned}
& \partial_{+}=\frac{\partial}{\partial x^{+}}=\frac{\alpha}{2}\left(\partial_{0}+\partial_{3}\right) \\
& \partial_{-}=\frac{\partial}{\partial x^{-}}=\frac{\alpha}{2}\left(\partial_{0}-\partial_{3}\right) \\
& \partial_{\perp}=\frac{\partial}{\partial x^{\perp}}
\end{aligned}
$$

Inverse differentiation is

$$
\begin{aligned}
& \partial_{0}=\frac{\partial}{\partial x^{0}}=\frac{1}{\alpha}\left(\partial_{+}+\partial_{-}\right) \\
& \partial_{3}=\frac{\partial}{\partial x^{3}}=\frac{1}{\alpha}\left(\partial_{+}-\partial_{-}\right)
\end{aligned}
$$

\subsection{Generalized light-front metric}

The metric tensor $g^{\mu \nu}$ connects contravariant and covariant tensor indices, a property we often refer to as the raising and lowering of tensor indices. So, for a 
given contravariant tensor, its covariant version can be determined straight away:

$$
A_{\mu}=g_{\mu \nu} A^{\nu}
$$

To determine the metric structure for the light front, we may consider the infinitesimal distance between two points in the Minkowski space-time, given by

$$
d s^{2}=\left(d x^{0}\right)^{2}-\left(d x^{1}\right)^{2}-\left(d x^{2}\right)^{2}-\left(d x^{3}\right)^{2},
$$

which, after transforming into light-front coordinates, we get

$$
d s^{2}=\alpha^{2} d x^{+} d x^{-}-\left(d x^{\perp}\right)^{2} .
$$

The most common normalization parameters that appear in the literature are $\alpha=1,2, \sqrt{2}$ for which we have, respectively,

$$
\begin{aligned}
& d s^{2}=d x^{+} d x^{-}-\left(d x^{\perp}\right)^{2}, \quad \alpha=1, \\
& d s^{2}=4 d x^{+} d x^{-}-\left(d x^{\perp}\right)^{2}, \quad \alpha=2, \\
& d s^{2}=2 d x^{+} d x^{-}-\left(d x^{\perp}\right)^{2}, \quad \alpha=\sqrt{2}
\end{aligned}
$$

We can now infer that the general light front metric tensor may be written as

$$
g_{\mu \nu}=\left(\begin{array}{cccc}
0 & \alpha^{2} / 2 & 0 & 0 \\
\alpha^{2} / 2 & 0 & 0 & 0 \\
0 & 0 & -1 & 0 \\
0 & 0 & 0 & -1
\end{array}\right)
$$

Thus, we have for

$$
\begin{aligned}
& x_{+}=g_{+\nu} x^{\nu}=g_{+-} x^{-} \\
& x_{-}=g_{-\nu} x^{\nu}=g_{-+} x^{+},
\end{aligned}
$$

the following results

$$
\begin{aligned}
& x_{+}=\frac{\alpha^{2}}{2} x^{-} \\
& x_{-}=\frac{\alpha^{2}}{2} x^{+} .
\end{aligned}
$$

For the tansverse components we have the expected change of signs

$$
x_{\perp}=g_{\perp \perp} x^{\perp}=-x^{\perp}
$$

\subsection{Generalized light-front: the Jacobian}

The Jacobian transformation for the generalized light front is given by

$$
|J|=\left|\begin{array}{cccc}
\frac{\alpha}{2} & \frac{\alpha}{2} & 0 & 0 \\
0 & 0 & 1 & 0 \\
0 & 0 & 0 & 1 \\
\frac{\alpha}{2} & -\frac{\alpha}{2} & 0 & 0
\end{array}\right|=\frac{\alpha^{2}}{2}
$$

where we used the expression for the Jacobian in Eq. (2) and the partial derivatives Eq. 24) and (25). For the common values of $\alpha=1,2, \sqrt{2}$, we have: $J=1 / 2,2,1$ respectively. These values has to be carefully laid down within integration measures.

\subsection{Integration in the light front}

To go from one coordinate system to another requires the use of Jacobian. Then, to go from the usual Minkowski coordinate system four-dimensional integration to the generalized light-front coordinate system integration we need

$$
\begin{aligned}
\int d x^{0} d x^{1} d x^{2} d x^{3} & =J \int d^{2} x^{\perp} d x^{+} d x^{-} \\
& =\frac{\alpha^{2}}{2} \int d^{2} x^{\perp} d x^{+} d x^{-}
\end{aligned}
$$

For the most common choices for the light front, $\alpha=1,2, \sqrt{2}$, we have, respectively:

$$
\int d x^{0} d x^{1} d x^{2} d x^{3}=\left\{\begin{array}{l}
\frac{1}{2} \int d^{2} x^{\perp} d x^{+} d x^{-} \\
2 \int d^{2} x^{\perp} d x^{+} d x^{-} \\
1 \int d^{2} x^{\perp} d x^{+} d x^{-}
\end{array}\right.
$$

\section{4. $\mathrm{D}^{\prime}$ Alambertian}

An application for 24, 25 and 29, is the scalar product:

$$
\begin{aligned}
\partial_{\mu} \partial^{\mu}= & g_{\mu \nu} \partial^{\mu} \partial^{\nu} \\
= & g_{+-} \partial^{+} \partial^{-}+g_{-+} \partial^{-} \partial^{+}+g_{++} \partial^{+} \partial^{+} \\
& +g_{--} \partial^{-} \partial^{-}+g_{\perp+} \partial^{\perp} \partial^{+} \\
& +g_{\perp-} \partial^{\perp} \partial^{-}+g_{+\perp} \partial^{+} \partial^{\perp}+g_{-\perp} \partial^{-} \partial^{\perp} \\
& +g_{\perp \perp} \partial^{\perp} \partial^{\perp} \\
\partial_{\mu} \partial^{\mu}= & \frac{\alpha^{2}}{2} \partial^{+} \partial^{-}+\frac{\alpha^{2}}{2} \partial^{+} \partial^{-}-\left(\partial^{\perp}\right)^{2},
\end{aligned}
$$

where $\alpha=1,2, \sqrt{2}$ for some of the most common normalization factors.In the light front, it can be obtained with the help of the generalized light front metric as follows

$$
\partial_{\mu} \partial^{\mu}=\alpha^{2} \partial^{+} \partial^{-}-\left(\partial^{\perp}\right)^{2}
$$

\section{Examples in the Light Front}

\subsection{Lorentz transformation}

Without loss of generality, as is common, we look at the Lorentz transformation for two inertial frame of 
references which move along the $z$ direction with respect to each other, for example,

$$
\left\{\begin{array}{l}
z^{\prime}=z \cosh \varphi-c t \sinh \varphi \\
c t^{\prime}=c t \cosh \varphi-z \sinh \varphi
\end{array}\right.
$$

where

$$
\varphi=\frac{1}{2} \ln \left(\frac{1+v / c}{1-v / c}\right)
$$

Substituting $z=x^{3}$ and $t=x^{0}$, with $c=1$ (in the natural units system), and using the definitions for the light front coordinates, we get the following system:

$$
\left\{\begin{array}{l}
x^{\prime+}=x^{+} \cosh \varphi-x^{+} \sinh \varphi \\
x^{\prime-}=x^{-} \cosh \varphi+x^{-} \sinh \varphi
\end{array}\right.
$$

The system in Eq. (39) is the Lorentz transformation in the Minkowski space-time expressed in terms of light front coordinates. We may simplify these noting that $\cosh \varphi \pm \sinh \varphi=\mathrm{e}^{ \pm \varphi}$ so that

$$
\begin{aligned}
& x^{{ }^{+}}=x^{+} e^{-\varphi} \\
& x^{\prime-}=x^{-} e^{\varphi}
\end{aligned}
$$

This result tells us that Lorentz transformations in the light front are very peculiar, behaving just like a kind of "scaling" factor. It is rather peculiar in that it does not mix different coordinates like it happens in the usual Minkowski space-time Lorentz transformation.

If the frame in movement is going in the opposite direction with respect to the "rest" frame, we use, as usual, the $-v$ for the relative velocity. This implies that Eq. (38) changes sign

$$
\varphi=\frac{1}{2} \ln \left(\frac{1+(-v / c)}{1-(-v / c)}\right)=-\frac{1}{2} \ln \left(\frac{1+v / c}{1-v / c}\right) .
$$

In the light front therefore, the equivalent to the frame of reference going into the opposite direction in relation to the "rest frame", i.e., $v<0$, is the change of sign in the argument of the exponential $\varphi$, which means Eq. 40. and Eq. 41) become:

$$
\begin{aligned}
& x^{++}=x^{+} e^{\varphi} \\
& x^{\prime-}=x^{-} e^{-\varphi}
\end{aligned}
$$

\subsection{Two successive Lorentz transformation in the light front}

Considering two parallel and consecutive Lorentz transformations between the referentials $R_{1}$ e $R_{2}$, moving away with constant speeds $v_{1}$ and $v_{2}$ in relation to the reference $R$. Two successive transformations in special relativity [6] we also have time dilation and space contraction for these moving systems of inertial frames, which are known to be given by

$$
\begin{aligned}
& \Delta t=\gamma_{2} \gamma_{1}\left(1+\frac{v_{1} v_{2}}{c^{2}}\right) \Delta t_{R} \\
& \Delta z=\frac{1}{\gamma_{1} \gamma_{2}}\left(\frac{1}{1+\frac{v_{1} v_{2}}{c^{2}}}\right) \Delta L_{R}
\end{aligned}
$$

in which we have introduced the proper time $\Delta t_{R}$ (time in the referential at rest) and proper length $\Delta L_{R}$ (length in the referential at rest) in the above formulae.

For $n$ successive transformations of the Lorentz transformation, we have:

$$
\begin{aligned}
& \Delta t=\prod_{i=1}^{n} \gamma_{i}\left(1+\frac{\prod_{i=1}^{n} v_{i}}{c^{2}}\right) \Delta t_{R} \\
& \Delta z=\frac{1}{\prod_{i=1}^{n} \gamma_{i}}\left(\frac{1}{1+\frac{\prod_{i=1}^{n} v_{i}}{c^{2}}}\right) \Delta L_{R}
\end{aligned}
$$

One particular interesting case occurs when the reference frames happen to be moving in opposite directions in relation to the one "at rest". In this case, Eq. (43) and Eq. (44), with for example $\vec{v}_{1}=-\vec{v}_{2}$, become

$$
\begin{aligned}
& \Delta t=\gamma_{2} \gamma_{1}\left(1-\frac{v_{1} v_{2}}{c^{2}}\right) \Delta t_{R} \\
& \Delta z=\frac{1}{\gamma_{1} \gamma_{2}}\left(\frac{1}{1-\frac{v_{1} v_{2}}{c^{2}}}\right) \Delta L_{R}
\end{aligned}
$$

In the special case $\vec{v}_{1}=-\vec{v}_{2}$ e $\left|\vec{v}_{1}\right|=\left|\vec{v}_{2}\right|=v$ 43, which implies in

$$
\Delta t=\Delta t_{R}
$$

If we consider two parallel and consecutive Lorentz transformations between reference frames $R_{2}$ and $R_{1}$, moving away with velocities $v_{2}$ and $v_{1}$ respectively in relation to a rest frame $R$, in the light-front we have:

$$
\begin{aligned}
& x^{+}=e^{-\left(\varphi_{1}+\varphi_{2}\right)} x_{R}^{+} \\
& x^{-}=e^{\left(\varphi_{1}+\varphi_{2}\right)} x_{R}^{-}
\end{aligned}
$$

where, of course, $\varphi$ is defined for each successive moving frame, using (38).

In general, in the ligth-front,

$$
\begin{aligned}
& x^{+}=e^{-\left(\sum_{i=1}^{\infty} \varphi_{i}\right)} x_{R}^{+}, \\
& x^{-}=e^{\left(\sum_{i=1}^{\infty} \varphi_{i}\right)} x_{R}^{-} .
\end{aligned}
$$

Now, because in the light front we have this peculiar form of Lorentz transformation that is tantamount as 
a scaling factor, an interesting particular case of three inertial frames of reference can be considered such that the first one we take as the "proper" inertial frame, that is, in which we have the proper time and proper length; the second one is the usual inertial frame that moves along the positive $z$ direction with velocity $v$ relative to the proper frame and the third one an inertial frame that moves along the negative $z$ direction with velocity $v$ relative to the first frame, Eq. (42). In such a particular case, we can see the same result of special relativity

$$
x^{+}=e^{-\left(\varphi_{1}+\varphi_{2}\right)} x_{R}^{+} .
$$

This happens because $\left|v_{1}\right|=\left|v_{2}\right|=v$ implies that $\left|\varphi_{1}\right|=$ $\left|\varphi_{2}\right|=\varphi$. However, for one of the reference frames going in the opposite direction, e.g., $v_{1}<0, \varphi<0$, Eq. 42 while $v_{2}>0, \varphi>0$ we conclude that

$$
x^{+}=x_{R}^{+}
$$

In this way, the light front is coherent with special relativity 48 .

\subsection{Canonical conjugate momentum in the light front}

In relativity, the mass squared is an invariant scalar which equals the four-momentum squared, that is

$$
k_{\mu} k^{\mu}=k^{2}=m^{2} .
$$

This entails an energy-momentum relation in the following manner

$$
\begin{aligned}
& k^{2}=k_{0}^{2}-\overrightarrow{\mathbf{k}}^{2}=m^{2} \\
& k_{0}^{2}=\overrightarrow{\mathbf{k}}^{2}+m^{2} \\
& k_{0}= \pm \sqrt{\overrightarrow{\mathbf{k}}^{2}+m^{2}}
\end{aligned}
$$

This quadratic relation that comes up between energy and three-momentum has been the source of difficulty until an adequate interpretation for the negative energy state could be given by Feynman. Moreover, in the quantum description of fields, both energy and momentum become hermitian operators, so that there is also this additional burden of defining what a square root of an operator might be [7].

The usual method of finding the energy ratio in the coordinates of the light front and calculating the scalar product $k^{\mu} k_{\mu}=m^{2}$ of the quadri-moment in these coordinates, with rest mass $m$. Therefore:

$$
k^{\mu} k_{\mu}=g_{\mu \nu} k^{\mu} k^{\nu}=\frac{\alpha^{2}}{2} k^{+} k^{-}+\frac{\alpha^{2}}{2} k^{-} k^{+}=m^{2}
$$

where we use 29 .

For the light front formulation, these difficulties are avoided simply because the energy momentum relation now reads

$$
k^{-}=\frac{\mathbf{k}_{\perp}^{2}+m^{2}}{\alpha^{2} k^{+}},
$$

which means that the sign for the light front energy $k^{-}$is always correlated to the sign of the longitudinal momentum $k^{+}$. Moreover, the relation between energy and momentum becomes linear, and so to speak like the usual non-relativistic quantum mechanics situation. Since this is so and knowing that in the usual nonrelativistic quantum mechanics the Schrödinger's equation is invariant under Galilean transformation, we were led to ask whether there could exist a similar situation or a situation in which one could mimic such a Galilean invariance in the transformations from one relativistic inertial frame of reference to another one [8]. And the answer becomes yes thanks to the structure of the Lorentz transformations when expressed in terms of light front coordinates.

\subsection{Schödinger equation: quantum propagator}

In Quantum Mechanics the wave function completely defines the state of the system and satisfies the Schrödinger equation [9, 10]:

$$
H|\Psi(t)\rangle=i \frac{d}{d t}|\Psi(t)\rangle,
$$

where $H$ is the Hamiltonian of the system

The wave function, $|\Psi(t)\rangle$, evolves over time and its evolution can be described by the unit operator $U\left(t-t^{\prime}\right)$ :

$$
|\Psi(t)\rangle=U\left(t-t^{\prime}\right)|\Psi(t)\rangle .
$$

The operator $U$ is unitary, because the evolution given by Eq. (54) preserve the probability

$$
\frac{d}{d t}\langle\Psi(t) \mid \Psi(t)\rangle=0
$$

Introducing the Eq. (55) in Eq. (54)we have:

$$
H U\left(t-t^{\prime}\right)=i \frac{d}{d t} U\left(t-t^{\prime}\right)
$$

where $U(0)=\mathbf{1}$.

Through the evolution operator, we can introduce the propagator of the Schröendiger equation

$$
S\left(t-t^{\prime}\right)=U\left(t-t^{\prime}\right) \theta\left(t-t^{\prime}\right),
$$

where $\theta(t)=1$ to $t>0$ and $t=0$ to $t<0$.

We conclude that the propagator is associated with the Green function of the Schroedinger equation as:

$$
G\left(t-t^{\prime}\right)=-i S\left(t-t^{\prime}\right)
$$

Green's function or the propagator completely describes the evolution of the quantum system. In this case we are using the spreader for "future times". We could also set the spreader to "back" in time.

We are going to study the spreader on a basis of $H$ autofunctions. The operator evolution and diagonal in this representation and its matriz element and a phase:

$$
U\left(t-t^{\prime}\right)=\sum\left|\Psi_{E}\right\rangle e^{-i E\left(t-t^{\prime}\right)}\left\langle\Psi_{E}\right|
$$

and where $H\left|\Psi_{E}\right\rangle=E\left|\Psi_{E}\right\rangle$ and $\hbar=1$. 
Thereby the propagator is given by:

$$
S\left(t-t^{\prime}\right)=\sum\left|\Psi_{E}\right\rangle e^{-i E\left(t-t^{\prime}\right)} \theta\left(t-t^{\prime}\right)\left\langle\Psi_{E}\right| .
$$

Defining the function $S_{E}(t)$ :

$$
S_{E}(t)=e^{-i E t} \theta(t) .
$$

The Fourier transform of $S_{E}(t)$ is:

$$
\begin{aligned}
S_{E}(\bar{E}) & =\frac{1}{\sqrt{2 \pi}} \int_{-\infty}^{+\infty} e^{i \bar{E} t} S_{E}(t) d t \\
& =\frac{1}{\sqrt{2 \pi}} \int_{0}^{+\infty} e^{i(\bar{E}-E) t} d t . \\
S_{E}(\bar{E}) & =\frac{i}{\sqrt{2 \pi}} \frac{1}{\bar{E}-E}
\end{aligned}
$$

where we use 11

$$
\int e^{a x} \cos (b x) d x=\frac{e^{a x}[a \cos (b x)+b \operatorname{sen}[b x]]}{a^{2}+b^{2}}
$$

Introducing the convergence factor, modifying $\bar{E} \rightarrow \bar{E}+i \varepsilon, \varepsilon>0$ :

$$
S_{E}(\bar{E})=\frac{i}{\sqrt{2 \pi}} \frac{1}{\bar{E}-E+i \varepsilon} .
$$

The contour condition corresponding to propagation to "Front" in time appears through the convergence factor $i \varepsilon$ in 63. Noting that,

$$
S_{E}(t)=\frac{1}{\sqrt{2 \pi}} \int_{-\infty}^{+\infty} d \bar{E} S_{E}(\bar{E}) e^{-i \bar{E} t} .
$$

We have that the propagator is given by:

$$
S\left(t-t^{\prime}\right)=\frac{i}{2 \pi} \int_{-\infty}^{+\infty} d \bar{E} \sum_{E}\left|\Psi_{E}\right\rangle \frac{e^{-i \bar{E}(t-t \prime)}}{\bar{E}-E+i \varepsilon}\left\langle\Psi_{E}\right| .
$$

Formally the Eq. 65 can be rewritten as:

$$
S\left(t-t^{\prime}\right)=\frac{i}{2 \pi} \int_{-\infty}^{+\infty} d \bar{E} e^{-i \bar{E}\left(t-t^{\prime}\right)} S_{F}(\bar{E}),
$$

where the Feynmam spreader is given by:

$$
S_{F}(E)=\frac{i}{E-H+i \varepsilon} .
$$

The great utility of Feynmam's idea in introducing propagators and in the construction of the perturbative series related to the breadth of a physical process.

\subsection{Klein-Gordon equation: quantum propagator}

Let us consider the Klein-Gordon equation with a source field,

$$
\left[\partial_{\mu} \partial^{\mu}+m^{2}\right] \psi(x)=\left[\left(\nabla^{2}-\frac{\partial^{2}}{\partial t^{2}}\right)+m^{2}\right] \psi(x)=-j(x)
$$

in which we are using $c=1$. A Green's function $G(x-y)$ associated to this equation is the solution of

$$
\left[\left(\nabla^{2}-\frac{\partial^{2}}{\partial t^{2}}\right)+m^{2}\right] G(x-y)=-\delta^{4}(x-y)
$$

Once the Green's function is determined, the solution for the original differential equation with source is given by

$$
\psi(x)=\int d^{4} y G(x-y) j(y)
$$

Finding the Green's function therefore is our first step to solve the problem of the field interaction with the source. As it is usual, we redefine the Green's function $G(x-y)$ by the propagator $S(x-y)$ in which $G(x-y)=$ $-i S(x-y)$. Then

$$
\left[\partial_{\mu} \partial^{\mu}+m^{2}\right] S(x-y)=-i \delta^{4}(x-y)
$$

where $\delta^{4}(x-y)=\int \frac{d^{4} k}{(2 \pi)^{4}} e^{-i k(x-y)}$.

Defining now the Fourier transform $S(x)$

$$
S\left(x^{\mu}\right)=\int \frac{d^{4} k}{(2 \pi)^{4}} S(k) e^{-i k x}
$$

By the standard resolution method putting Eq. 71 into Eq. (70) we get:

$$
S(k)=\frac{i}{k^{2}-m^{2}+i \epsilon}
$$

This, of course, is the covariant Feynman propagator for the Klein-Gordon field, where the infinitesimal imaginary part $i \epsilon$ is chosen in such a way so as to guarantee time evolution of the system into the future. From Eq. 72 we observe that in the complex $k^{0}$ plane energy has two simple poles, one for each of the two solutions for the energy. Thus, we can interpret it as two propagations with positive energy, one forward in time $t$ and the other one backward in time $t$. Of course, physically speaking this does not make sense, so Feynman interpreted it as an antiparticle traveling forward in time $t$ [4].

\subsection{Klein-Gordon equation: quantum propagator in the light-front}

Using the coordinate transformation properties for the light front, in 71 , for the special choice of $\alpha=\sqrt{2}$ which for us is the most convenient choice because the Jacobian of the transformation, $|J|=\frac{\alpha^{2}}{2}$, turns out to be unit, we have

$$
S\left(x^{\mu}\right)=\int \frac{d k^{-} d k^{+} d^{2} k^{\perp}}{(2 \pi)^{4}} S(k) e^{-i\left[k^{-} x^{+}+k^{+} x^{-}-k^{\perp} x^{\perp}\right]} .
$$


Since Eq. 70 is built supposing a source, it means that for the light front case we have

$$
\begin{aligned}
& {\left[2 \partial^{+} \partial^{-}-\left(\partial^{\perp}\right)^{2}+m^{2}\right]} \\
& \qquad \frac{d k^{-} d k^{+} d^{2} k^{\perp}}{(2 \pi)^{4}} S(k) e^{-i\left[k^{-} x^{+}+k^{+} x^{-}-k^{\perp} x^{\perp}\right]} \\
& \quad=-i \int \frac{d k^{-} d k^{+} d^{2} k^{\perp}}{(2 \pi)^{4}} e^{-i\left[k^{-} x^{+}+k^{+} x^{-}-k^{\perp} x^{\perp}\right]}
\end{aligned}
$$

or

$$
\begin{aligned}
\int \frac{d k^{-} d k^{+} d^{2} k^{\perp}}{(2 \pi)^{4}} S(k)\left[2 \partial^{+} \partial^{-}-\left(\partial^{\perp}\right)^{2}+m^{2}\right] \\
e^{-i\left[k^{-} x^{+}+k^{+} x^{-}-k^{\perp} x^{\perp}\right]} \\
=-i \int \frac{d k^{-} d k^{+} d^{2} k^{\perp}}{(2 \pi)^{4}} e^{-i\left[k^{-} x^{+}+k^{+} x^{-}-k^{\perp} x^{\perp}\right]}
\end{aligned}
$$

consequently

$$
\begin{aligned}
\int \frac{d k^{-} d k^{+} d^{2} k^{\perp}}{(2 \pi)^{4}} S(k)\left[-2 k^{+} k^{-}+\left(k^{\perp}\right)^{2}+m^{2}\right] \\
e^{-i\left[k^{-} x^{+}+k^{+} x^{-}-k^{\perp} x^{\perp}\right]} \\
=-i \int \frac{d k^{-} d k^{+} d^{2} k^{\perp}}{(2 \pi)^{4}} e^{-i\left[k^{-} x^{+}+k^{+} x^{-}-k^{\perp} x^{\perp}\right]}
\end{aligned}
$$

this equality is only verified if the integrand are equal, i.e :

$$
S(k)\left[-2 k^{+} k^{-}+\left(k^{\perp}\right)^{2}+m^{2}\right]=-i
$$

result

$$
S_{\mathrm{LF}}(k)=\frac{i}{2 k^{+}\left[k^{-}-\frac{k^{\perp^{2}+m^{2}}}{2 k^{+}}+\frac{i \epsilon}{2 k^{+}}\right]}
$$

This last Eq. (74) is the Klein-Gordon field propagator in the light front. Here, because there is a linear relation between the light front energy $k^{-}$and the longitudinal momentum component $k^{+}$, particle and/or antiparticle propagation depends on the choice for the sign of the longotudinal momentum. We can interpret the simple pole $k^{-}=\frac{k^{\perp^{2}}+m^{2}}{2 k^{+}}$as the energy that the particle possess. That means that describing a relativistic particle propagating forward in the light front time is tantamount to a propagation of the particle in the non relativistic quantum mechanics (67) [8, 12,16.

\section{Conclusions}

Using the defiition for the coordinate transformation to the light front, we deduced the Lorentz transformations in the light front, were we applied this to analyse Lorentz transformation for three particular inertial reference frames. We also obtained the general metric in the light front gauge and the form of its internal (scalar) product.

With these results we applied to the case of the KleinGordon field to illustrate how the relativistic quantum propagator for particles with spin 1 (bosons) can be obtained in the light front.

The advantage of using the quantum propagator in the light front is that its description is similar to the non relativistic quantum mechanics. In this sense at least in principle - the quantum field formulation would be simpler. This is the very fact that have encouraged many to apply this formulation in the light front for more complex cases like gauge fields in quantum electrodynamics [17, 18] and quantum chromodynamics.

\section{Acknowledgments}

JHS thanks CNPq, FAPESB and CAPES; DNP thanks FAPESB for support and GSS thanks IFBA.

\section{REFERENCES}

[1] H. Minkowski, Jahresbericht der Deutschen Mathematiker-Vereinigung 18, 75 (1909).

[2] P.A.M. Dirac, Rev. Mod.Phys. 21, 392 (1949).

[3] J.H.O. Sales and A.T. Suzuki, Communications in Theoretical Physics 60, 55 (2013).

[4] J.H. Sales, A.T. Suzuki and L.A. Soriano, Revista Brasileira de Ensino de Física 37, 3309 (2015).

[5] J.H. Sales, A.T. Suzuki, D.N. Possidonio, I.G. Oliveira and P.H. Girotto, Revista Brasileira de Ensino de Física 42, e20190290 (2020).

[6] J. Loureiro, Física Relativística (Editora IST Press, Lisboa 2008).

[7] J.H.O. Sales, T. Frederico, B.V. Carlson and P.U. Sauer, Phys. Rev. 61, 044003 (2000).

[8] S.J. Brodsky, H.C. Pauli and S. Pinski, Phys. Rep. 301, 299 (1998).

[9] A. Messiah, Quantum Mechanics (Dover Publications, Mineola, 1999).

[10] J.J.J. Sakurai and J. Napolitano, Modern Quantum Mechanics (Addison Wesley Publishing Company Incorporated, Boston, 2010).

[11] M.R. Spiegel and R. Murray, Manual de Fórmulas e Tabelas Matemáticas (Bookman, Porto Alegre, 2004).

[12] J.H.O. Sales and A.T. Suzuki, Int J Theor Phys 48, 2340 (2009).

[13] J.H. Sales, A.T. Suzuki and G.S. Santos, Few-Body Systems 56, 587 (2015).

[14] J.H. Sales, A.T. Suzuki, G.S. Santos and C.A. Lima, Few-Body Systems 56, 593 (2015).

[15] A.T. Suzuki, J.H. Sales and G.S. Santos, Few-Body Systems 56, 599 (2015).

[16] J.H.O. Sales and A.T.Suzuki, Commun. Theor. Phys. 55, 1029 (2011).

[17] I.G. Oliveira, J.H. Sales and R. Thibes, Eur. Phys. J. Plus 135, 713 (2020).

[18] C.R. Ji, A. Takashi Suzuki, H. Sales and J. Thibes, R. Eur. Phys. J. 79, 871 (2019). 